\title{
MIXED MULTIPLICITIES, JOINT REDUCTIONS AND QUASI-UNMIXED LOCAL RINGS
}

\author{
IRENA SWANSON
}

\section{Introduction}

Joint reductions and mixed multiplicities are relatively new concepts and there is very little about them in the literature. We provide some background in the first two sections. Some of the properties about joint reductions and mixed multiplicities which we prove have not appeared elsewhere, but are probably folklore to the people who have worked on mixed multiplicities or joint reductions. This makes it very difficult to attribute them to their rightful discoverers.

The story of mixed multiplicities starts in 1957 with Bhattacharya's paper [1], where he described Hilbert polynomials for two ideals and their highest degree coefficients. The name mixed multiplicities first appeared in Teissier's paper [15], published in 1973, in which he and Risler studied Hilbert polynomials and their coefficients for any finite number of ideals by using superficial elements (of a then new kind). The connection between mixed multiplicities and joint reductions was first observed by Rees in 1983 during a research symposium associated with the Nordic Summer School. This was also the first time that joint reductions were defined. Rees' approach to mixed multiplicities, as displayed in his 1984 paper [11] and later in his book [13], is different from Teissier's; Rees studies mixed multiplicities via multiplicities of certain ideals in 'general' extensions of the original ring. Other authors have used Rees algebras and blow-ups for studying joint reductions and mixed multiplicities (see for example $[3,17]$ ).

A motivation for this work was Rees' multiplicity theorem (cf. [11, Corollary 3.8]) which relates multiplicities to reductions in quasi-unmixed local rings. A similar relation holds between mixed multiplicities and joint reductions, and that is the main theorem of this paper (Theorem 3.7). I am indebted to Jugal Verma for suggesting this problem and for supplying a proof in dimension 2 (see [16]). Rees' original theorem and Böger's generalization of it [2, Corollary 3.9] both follow from Theorem 3.7. For very different proofs of Rees' multiplicity theorem see also Rees and Sharp [14], Katz [3] and Kirby and Rees [4]. Kirby and Rees use Buchsbaum-Rim multiplicities and an elaboration of Rees' machinery from [12]. Kirby communicated to me that in a future version of [4] there will be a generalization of Theorem 3.7 of this paper.

Section 1 gives the definition of joint reductions. The original concept of joint reductions, given by Rees in [12], was defined for $d m$-primary ideals in a local ring $(R, m)$ of dimension $d$. O'Carroll's and Verma's definitions of joint reductions [8 and 18] are not as restrictive, and the definition of joint reductions in Section 1 here works in even more general settings. Section 1 also gives the machinery which is necessary for the main results. This machinery comes mostly from Teissier's rich paper [15]. Section 2 proves a few lemmas about joint reductions and mixed multiplicities. Some

Received 21 November 1991.

1991 Mathematics Subject Classification 13H15.

J. London Math. Soc. (2) 48 (1993) 1-14 
of these were previously proved by Rees using the theory of general elements [13]. The proofs given here do not use general elements. Section 3 contains the main results, in particular Theorem 3.7. Some corollaries of this result are Rees' and Böger's multiplicity theorems.

\section{Definitions}

Definition 1.1 (cf. Northcott-Rees [7]). Let $I$ and $J$ be ideals in $R, I \subseteq J$. Then $I$ is a reduction of $J$ if there exists an integer $l$ such that $J^{l}=I J^{l-1}$.

Note that in this case $J^{m+n}=I^{m} J^{n}$ for all $m \geqslant 1$ and all $n \geqslant l-1$.

Definition 1.2. Let $R$ be a Noetherian ring and $q_{1}, \ldots, q_{k}$ ideals in $R$. Let $a_{i} \in q_{i}$. Then the $k$-tuple $\left(a_{1}, \ldots, a_{k}\right)$ is called a joint reduction of $\left(q_{1}, \ldots, q_{k}\right)$ if $a_{1} q_{2} \ldots q_{k}+\ldots+a_{k} q_{1} \ldots q_{k-1}$ is a reduction of the ideal $q_{1} \ldots q_{k}$, that is if there exists an integer $l$ such that

$$
\left(q_{1} \ldots q_{k}\right)^{l}=a_{1} q_{1}^{l-1} q_{2}^{l} \ldots q_{k}^{l}+\ldots+a_{k} q_{1}^{l} \ldots q_{k-1}^{l} q_{k}^{l-1} .
$$

If $M$ is an $R$-module and $\left(q_{1} \ldots q_{k}\right)^{l} M=a_{1} q_{1}^{l-1} q_{2}^{l} \ldots q_{k}^{l} M+\ldots+a_{k} q_{1}^{l} \ldots q_{k-1}^{l} q_{k}^{l-1} M$, then we say that $\left(a_{1}, \ldots, a_{k}\right)$ is a joint reduction of $\left(q_{1}, \ldots, q_{k}\right)$ with respect to $M$.

EXAMPLE 1.3. Let $R=k[X, Y]$. Then $(X, Y)$ is a joint reduction of $\left(\left(X, Y^{n}\right),\left(X^{m}, Y\right)\right)$ for all $n, m \geqslant 1$, as $X\left(X^{m}, Y\right)+Y\left(X, Y^{n}\right)=\left(X, Y^{n}\right)\left(X^{m}, Y\right)$.

REMARK 1.4. By writing out the ideal equalities it is easy to see that the $k$-tuple $\left(a_{1}, \ldots, a_{k}\right)$ is a joint reduction of $(q, \ldots, q)$ (with respect to $\left.M\right)$ if and only if the ideal $\left(a_{1}, \ldots, a_{k}\right)$ is a reduction of $q$ (with respect to $M$ ).

The context should make it clear when $\left(a_{1}, \ldots, a_{k}\right)$ is to be thought of as a $k$-tuple and when as an ideal.

DeFINITION 1.5. If $(R, m)$ is a Noetherian local ring and $I$ is an $m$-primary ideal, then $e_{I}(M)$ denotes the multiplicity of $M$ with respect to $I$. If $M=R$, then $e_{I}(R)$ is also called the multiplicity of $I$. Sometimes we shall write $e(I ; M)$ instead of $e_{I}(M)$.

Note that if $I$ and $J$ are ideals such that $J \subseteq$ ann $(M)$ and $I+J$ is $m$-primary, then $\lambda\left(M / I^{n} M\right)=\lambda\left(M /(I+J)^{n} M\right)$ for all $n$, so we may write $e_{I}(M)$ instead of $e_{I+J}(M)$.

Definition 1.6. A sufficiently general element of an ideal $I$ means the following. Let $I$ be minimally generated by $l$ elements, say $x_{1}, \ldots, x_{l}$. Suppose that there exists a non-empty Zariski-open subset $U$ of $k^{l}$ such that whenever $a=\sum y_{t} x_{t}$ and the image of $\left(y_{1}, \ldots, y_{l}\right)$ in $k^{l}$ lies in $U$, then $a$ satisfies a given property. Then that property is called sufficiently general and any such $a$ is called a sufficiently general element of $I$ (with respect to that property).

The advantage of sufficiently general properties is that whenever $k$ is infinite, it is possible to find an element satisfying finitely many sufficiently general properties, for the intersection of finitely many non-empty Zariski-open subsets is still non-empty and Zariski-open.

Teissier proved the following theorem. 
THEOREM 1.7 [15]. Let $(R, m)$ be a Noetherian local ring. Suppose that $q_{1}, \ldots, q_{k}$ are $m$-primary ideals and that $M$ is a finitely generated $R$-module. Then there exists an integer $c \geqslant 0$ such that

$$
\lambda\left(M / q_{1}^{n_{1}} \ldots q_{k}^{n_{k}} M\right)
$$

is a polynomial in $n_{1}, \ldots, n_{k}$ of degree $\operatorname{dim}(M)$ if $n_{i} \geqslant c$ for each $i, 1 \leqslant i \leqslant k$.

The homogeneous polynomial of degree $\operatorname{dim}(M)$ in $\lambda\left(M / \underline{q}^{\underline{n}} M\right)$ can be written as

$$
\sum_{d_{1}+\ldots+d_{k} \text {-dim }(M)} \frac{1}{d_{1} ! \ldots d_{k} !}\left[q_{1}^{\left[d_{1}\right]}, \ldots, q_{k}^{\left[d_{k}\right]} ; M\right] n_{1}^{d_{1}} \ldots n_{k}^{d_{k}},
$$

where $\left[q_{1}^{\left[d_{1}\right]}, \ldots, q_{k}^{\left[d_{k}\right]} ; M\right]$ is a positive integer.

Definition 1.8. The integer $\left[q_{1}^{\left[d_{1}\right]}, \ldots, q_{k}^{\left[d_{k}\right]} ; M\right]$ is called the mixed multiplicity of the module $M$ of type $\left(d_{1}, \ldots, d_{k}\right)$ with respect to the ideals $q_{1}, \ldots, q_{k}$.

An important ingredient in Teissier's proof is the following lemma.

LEMMA 1.9. Let $(R, m, k)$ be a Noetherian local ring and suppose that the residue field $k$ is infinite. Let $q_{1}, \ldots, q_{k}$ be ideals in $R$ and $M$ a finitely-generated $R$-module. Let $\left\{P_{1}, \ldots, P_{s}\right\}$ be a set of primes not containing $q_{1}$. Then there exist a sufficiently general element $a$ in $q_{1}$ which does not lie in any $P_{i}$, and an integer $c_{0} \geqslant 0$, such that for each $i \geqslant 1$,

$$
\left(\underline{q}^{\underline{n}} M:{ }_{M} a^{i}\right) \bigcap q_{1}^{c} q_{2}^{n_{2}} \ldots q_{k}^{n_{k}} M=q_{1}^{n_{1}-i} q_{2}^{n_{8}} \ldots q_{k}^{n_{k}} M \text { for all } n_{2}, \ldots, n_{k},
$$

whenever $c \geqslant c_{0}$ and $n_{1} \geqslant c+i$.

This lemma gives rise to the usual definition of superficial elements. However, in the proof of the main theorem we need a more general definition.

Definition 1.10 . Let $R$ be a Noetherian ring, not necessarily local. Let $q_{1}, \ldots, q_{k}$ be ideals of $R$ and let $M$ be a finitely-generated $R$-module. Let $a$ be an element of $q_{1}$ such that $\operatorname{dim}(M / a M)=\operatorname{dim}(M)-1$ and such that

$$
\left(q^{n} M:{ }_{M} a\right) \bigcap q_{1}^{c} q_{2}^{n_{2}} \ldots q_{k}^{n_{k}} M=q_{1}^{n_{1}-1} q_{2}^{n_{2}} \ldots q_{k}^{n_{k}} M
$$

for all sufficiently large $n_{1}$ and all $n_{2}, \ldots, n_{k}$. Then $a$ is called superficial for $q_{1}, \ldots, q_{k}$ and $M$.

Teissier also showed the following.

THEOREM 1.11. If $R$ has infinite residue field, and $a_{1}, \ldots, a_{d}$ are elements of $R$, the first $d_{1}$ of which are elements of $q_{1}, \ldots$, and the last $d_{k}$ of which are elements of $q_{k}$, such that $a_{1}$ is superficial for $q_{1}, \ldots, q_{k}$ and $M, a_{2}$ is superficial for $q_{2}, q_{1}, \ldots, q_{k}$ and $M / a_{1} M$, etc., then

$$
\begin{aligned}
{\left[q_{1}^{\left[d_{1}\right]}, \ldots, q_{k}^{\left[d_{k}\right]} ; M\right] } & =\left[q_{1}^{\left[d_{1}-1\right]}, q_{2}^{\left[d_{2}\right]}, \ldots, q_{k}^{\left[d_{k}\right]} ; M / a_{1} M\right] \\
& =\ldots \\
& =\lambda\left(M /\left(a_{1}, \ldots, a_{d}\right) M\right)-\lambda\left(0:_{M /\left(a_{1}, \ldots, a_{d-1}\right) M} a_{d}\right),
\end{aligned}
$$

which equals $e_{\left(a_{1}, \ldots, a_{d}\right)}(M)$. In other words, $\left[q_{1}^{\left[d_{1}\right]}, \ldots, q_{k}^{\left[d_{k}\right]} ; M\right]$ equals the multiplicity of any ideal generated by a superficial sequence composed of $d_{1}$ elements of $q_{1}$, etc., and $d_{k}$ elements of $q_{k}$. 
It turns out that such a superficial sequence forms a joint reduction of the $d$-tuple $\left(q_{1}, \ldots, q_{1}, \ldots, q_{k}, \ldots, q_{k}\right)$ with respect to $M$, where each $q_{i}$ appears $d_{i}$ times. Thus we also get the existence of joint reductions of $d m$-primary ideals with respect to $M$.

Northcott and Rees proved in [7] that $e_{I}(R)=e_{J}(R)$ if $I$ is a reduction of $J$. The analogous statement for mixed multiplicities and joint reductions was proved by Rees. Namely, Rees proved in [12, Theorem 2.4] that the number $e\left(\left(a_{1}, \ldots, a_{d}\right) ; M\right)$ is the same for every joint reduction $\left(a_{1}, \ldots, a_{d}\right)$ of $\left(q_{1}, \ldots, q_{1}, \ldots, q_{k}\right)$ with respect to $M$, where each $q_{i}$ appears $d_{i}$ times. Since superficial sequences generate a joint reduction, it follows that $\left[q_{1}^{\left[d_{1}\right]}, \ldots, q_{k}^{\left[d_{k}\right]} ; M\right]=e\left(\left(a_{1}, \ldots, a_{d}\right) ; M\right)$ for every joint reduction $\left(a_{1}, \ldots, a_{d}\right)$ of $\left(q_{1}, \ldots, q_{1}, \ldots, q_{k}\right)$ with respect to $M$. Thus

$$
\left[q_{1}^{\left[d_{1}\right]}, \ldots, q_{k}^{\left[d_{k}\right]} ; M\right]=[\underbrace{q_{1}^{[1]}, \ldots, q_{1}^{[1]}}_{d_{1} \text { times }}, \ldots, \underbrace{q_{k}^{[1]}, \ldots, q_{k}^{[1]}}_{d_{k} \text { times }} ; M],
$$

so it is only necessary to analyze mixed multiplicities of type $(1, \ldots, 1)$. Rees introduced the following notation for such mixed multiplicities:

$$
e\left(q_{1}, \ldots, q_{d} ; M\right)=\left[q_{1}^{[1]}, \ldots, q_{d}^{[1]} ; M\right] .
$$

Thus without loss of generality we may use only mixed multiplicities of type $(1, \ldots, 1)$.

\section{Properties of joint reductions and mixed multiplicities}

In this section we prove several lemmas and propositions about joint reductions and mixed multiplicities which are needed for the proof of the main theorem.

LEMMA 2.1. Let $R$ be a Noetherian ring (not necessarily local), $q_{1}, \ldots, q_{k}$ ideals of $R$ and $a_{i} \in q_{i}$ for $i=1, \ldots, k$. Then the following statements are equivalent:

(i) $\left(a_{1}, \ldots, a_{k}\right)$ is a joint reduction of $\left(q_{1}, \ldots, q_{k}\right)$;

(ii) for all positive integers $l_{1}, \ldots, l_{k},\left(a_{1}^{l_{1}}, \ldots, a_{k}^{l_{k}}\right)$ is a joint reduction of $\left(q_{1}^{l_{1}}, \ldots, q_{k}^{l_{k}}\right)$;

(iii) for some positive integers $l_{1}, \ldots, l_{k},\left(q_{1}^{l_{1}}, \ldots, a_{k}^{l_{k}}\right)$ is a joint reduction of $\left(q_{1}^{l_{1}}, \ldots, q_{k}^{l_{k}}\right)$.

Proof. Assume that $\left(a_{1}, \ldots, a_{k}\right)$ is a joint reduction of $\left(q_{1}, \ldots, q_{k}\right)$. In order to prove the second condition, it suffices to show that for any positive integer $n$, $\left(a_{1}^{n}, a_{2}, \ldots, a_{k}\right)$ is a joint reduction of $\left(q_{1}^{n}, q_{2}, \ldots, q_{k}\right)$. We use induction on $n$. If $n=1$, there is nothing to show. So assume that $n>1$. By the inductive hypothesis there exists a positive integer $l$ such that

$$
\begin{aligned}
\left(q_{1} \ldots q_{k}\right)^{l} & =a_{1} q_{1}^{l-1} q_{2}^{l} \ldots q_{k}^{l}+\ldots+a_{k} q_{1}^{l} \ldots q_{k-1}^{l} q_{k}^{l-1} \\
\left(q_{1}^{n-1} q_{2} \ldots q_{k}\right)^{l} & =a_{1}^{n-1} q_{1}^{(n-1)(l-1)} q_{2}^{l} \ldots q_{k}^{l}+\ldots+a_{k} q_{1}^{(n-1) l} q_{2}^{l} \ldots q_{k-1}^{l} q_{k}^{l-1} .
\end{aligned}
$$

Then

$$
\begin{aligned}
\left(q_{1}^{n} q_{2} \ldots q_{k}\right)^{l}= & q_{1}^{l}\left(q_{1}^{n-1} q_{2} \ldots q_{k}\right)^{l} \\
= & q_{1}^{l}\left[a_{1}^{n-1} q_{1}^{(n-1)(l-1)} q_{2}^{l} \ldots q_{k}^{l}+\ldots+a_{k} q_{1}^{(n-1) l} q_{2}^{l} \ldots q_{k-1}^{l} q_{k}^{l-1}\right] \\
= & a_{1}^{n-1} q_{1}^{(n-1)(l-1)+l} q_{2}^{l} \ldots q_{k}^{l}+\ldots+a_{k} q_{1}^{n l} q_{2}^{l} \ldots q_{k-1}^{l} q_{k}^{l-1} \\
\subseteq & a_{1}^{n-1} q_{1}^{(n-1)(l-1)}\left[a_{1} q_{1}^{l-1} q_{2}^{l} \ldots q_{k}^{l}+\ldots+a_{k} q_{1}^{l} \ldots q_{k-1}^{l} q_{k}^{l-1}\right] \\
& +\ldots+a_{k} q_{1}^{n l} q_{2}^{l} \ldots q_{k-1}^{l} q_{k}^{l-1} \\
\subseteq & a_{1}^{n} q_{1}^{n(l-1)} q_{2}^{l} \ldots q_{k}^{l}+\ldots+a_{k} q_{1}^{n l} q_{2}^{l} \ldots q_{k-1}^{l} q_{k}^{l-1}
\end{aligned}
$$


Conversely, if $\left(a_{1}^{l_{1}}, \ldots, a_{k}^{l_{k}}\right)$ is a joint reduction of $\left(q_{1}^{l_{1}}, \ldots, q_{k}^{l_{k}}\right)$ for some positive integers $l_{1}, \ldots, l_{k}$, then there exists a positive integer $l$ such that

$$
\left(q_{1}^{l_{1}} \ldots q_{k}^{l_{k}}\right)^{l}=a_{1}^{l_{1}} q_{1}^{l_{1}(l-1)} q_{2}^{l_{2} l} \ldots q_{k}^{l_{k} l}+\ldots+a_{k}^{l_{k}} q_{1}^{l_{1} l} \ldots q_{k-1}^{l_{k}-1} q_{k}^{l_{k}(l-1)} .
$$

But the latter ideal is contained in

$$
a_{1} q_{1}^{l_{1} l-1} q_{2}^{l_{2} l} \ldots q_{k}^{l_{k} l}+\ldots+a_{k} q_{1}^{l_{1} l} \ldots q_{k-1}^{l_{k-1} l} q_{k}^{l_{k} l-1}
$$

which says that $\left(a_{1}, \ldots, a_{k}\right)$ is a joint reduction of $\left(q_{1}, \ldots, q_{k}\right)$.

LEMMA 2.2. Let $R$ be a Noetherian ring, $q_{1}, \ldots, q_{k}$ ideals and $a_{i} \in q_{i}$. Then there exist ideals $p_{i} \subseteq q_{i}$ such that $a_{i} \in p_{i}$ and such that $\left(a_{1}, \ldots, a_{k}\right)$ is a joint reduction of $\left(p_{1}, \ldots, p_{k}\right)$.

Moreover, if $\left(a_{1}, \ldots, a_{k}\right)$ and each $q_{i}$ is primary to the same maximal ideal of $R$, we may choose the $p_{i}$ to be primary to the same maximal ideal.

Proof. For each $i=1, \ldots, k$, define $p_{i}=\left(a_{1}\right) \cap q_{i}+\ldots+\left(a_{k}\right) \cap q_{i}$. So $p_{i} \subseteq q_{i}$. We shall show that

$$
p_{1} \ldots p_{k} \subseteq a_{1} p_{2} \ldots p_{k}+\ldots+a_{k} p_{1} \ldots p_{k-1},
$$

which will finish the proof of the first part. The second part follows by the construction of the $p_{i}$.

If $k=1, p_{1}=\left(a_{1}\right)$, so clearly $\left(a_{1}\right)$ is a joint reduction of $p_{1}$. Now assume that $k>1$. For $i=1, \ldots, k$, choose $b_{i} \in p_{i}$, so $b_{i}=\sum_{j-1}^{k} r_{i j} a_{j}$, where each $r_{i j} a_{j} \in\left(a_{j}\right) \cap q_{i} \subseteq p_{i}$. Then $b_{1} \ldots b_{k}$ is the sum of the terms of the form

$$
T=\left(r_{1 j_{1}} a_{j_{1}}\right) \ldots\left(r_{k j_{k}} a_{j_{k}}\right) .
$$

If all the $j_{i}$ are distinct, then $T$ is contained in $\left(a_{1} \ldots a_{k}\right) \subseteq a_{1} p_{2} \ldots p_{k}$, so we are done. But if the $j_{i}$ are not distinct, then one of the $a_{i}$ does not appear, say $a_{k}$. Let $p_{i}^{\prime}=\left(a_{1}\right) \cap q_{i}+\ldots+\left(a_{k-1}\right) \cap q_{i}$ for each $i$. Since for $i<k$,

then by induction

$$
r_{i j_{i}} a_{j_{i}} \in\left(a_{1}\right) \cap q_{i}+\ldots+\left(a_{k-1}\right) \cap q_{i}=p_{i}^{\prime},
$$

$$
\begin{aligned}
T^{\prime} & =\left(r_{1 j_{1}} a_{j_{1}}\right) \ldots\left(r_{k-1, j_{k-1}} a_{j_{k-1}}\right) \in a_{1} p_{2}^{\prime} \ldots p_{k-1}^{\prime}+\ldots+a_{k-1} p_{1}^{\prime} \ldots p_{k-2}^{\prime} \\
& \subseteq a_{1} p_{2} \ldots p_{k-1}+\ldots+a_{k-1} p_{1} \ldots p_{k-2} .
\end{aligned}
$$

Hence

$$
T=T^{\prime}\left(r_{k j_{k}} a_{j_{k}}\right) \in\left(a_{1} p_{2} \ldots p_{k-1}+\ldots+a_{k-1} p_{1} \ldots p_{k-2}\right) p_{k} .
$$

Lemma 2.3. Let $(R, m)$ be a Noetherian local ring. Set $S=R[X]_{m R[X]}$, where $X$ is an indeterminate over $R$. Let $M$ be a finitely generated $R$-module of dimension d and let $q_{1}, \ldots, q_{d}$ be m-primary ideals. Then

$$
e\left(q_{1}, \ldots, q_{d} ; M\right)=e\left(q_{1}, S, \ldots, q_{d} S ; M S\right) .
$$

Proof. It is easy to see that $m S$ is the maximal ideal of $S$ and that $q_{i} S$ is $m S$-primary. So $M S / \underline{q}^{\underline{n}} M S$ has finite length whenever some $n_{i}$ is positive. But $\lambda_{S}\left(M S / \underline{q}^{\underline{n}} M S\right)=\lambda_{R}\left(M / \underline{q}^{\underline{n}} M\right)$, so the two polynomials describing these quantities for sufficiently large $\underline{n}$ are equal. 
LEMMA $2.4[13$, p. 184]. Let $(R, m)$ be a Noetherian local ring, and $M$ a finitely generated $R$-module of dimension $d$. Let $p_{i} \subseteq q_{i}$ be m-primary ideals for $i=1, \ldots, d$. Then $e\left(p_{1}, \ldots, p_{d} ; M\right) \geqslant e\left(q_{1}, \ldots, q_{d} ; M\right)$.

Proof. It is easy to see that it is enough to prove the statement for the case when $p_{1}=q_{1}, \ldots, p_{d-1}=q_{d-1}$ and $p_{d} \subseteq q_{d}$.

If $d=1$, then $\lambda\left(M / p_{1}^{n} M\right) \geqslant \lambda\left(M / q_{1}^{n} M\right)$, so necessarily $e\left(p_{1} ; M\right) \geqslant e\left(q_{1} ; M\right)$.

Now let $d>1$. By Lemma 2.3 we may assume that $R$ has an infinite residue field. Then by Theorem 1.11 there exists an element $a \in p_{1}=q_{1}$ such that $\operatorname{dim}(M / a M)=\operatorname{dim}(M)-1$ and

$$
\begin{gathered}
e\left(p_{1}, \ldots, p_{d} ; M\right)=e\left(p_{2}, \ldots, p_{d} ; M / a M\right) \\
e\left(q_{1}, \ldots, q_{d} ; M\right)=e\left(q_{2}, \ldots, q_{d} ; M / a M\right)
\end{gathered}
$$

So by induction on $d$ we are done.

LEMMA 2.5. If $(R, m)$ is a local ring, $M$ a finitely generated $R$-module and $q$ an m-primary ideal, then $e_{q}(M)=e(q, \ldots, q ; M)$.

Proof. By Lemma 2.3 we may assume that $R$ has an infinite residue field. With this the proof follows easily: since the residue field of $R$ is infinite, by Theorem 1.11 there exists a superficial sequence $a_{1}, \ldots, a_{d}$ in $q$ (of type $(d)$ ) such that by Theorem 1.11 ,

$$
e\left(\left(a_{1}, \ldots, a_{d}\right) ; M\right)=e(q, \ldots, q ; M)
$$

But the $d$-tuple $\left(a_{1}, \ldots, a_{d}\right)$ is a joint reduction of $(q, \ldots, q)$, so the ideal $\left(a_{1}, \ldots, a_{d}\right)$ is a reduction of $q$, hence $e_{q}(M)=e\left(\left(a_{1}, \ldots, a_{d}\right) ; M\right)$.

LEMMA 2.6. Let $M$ be a finitely generated $R$-module of dimension $d, q_{1}, \ldots, q_{d}$ $m$-primary ideals and $l_{1} \ldots, l_{d}$ positive integers. Then

$$
e\left(q_{1}^{l_{1}}, \ldots, q_{d}^{l_{d}} ; M\right)=l_{1}^{a_{1}} \ldots l_{k}^{a_{k}} e\left(q_{1}, \ldots, q_{d} ; M\right)
$$

Proof. Just look at the coefficients of the polynomial $\lambda\left(M / \underline{q}^{\underline{n}} M\right)$.

Proposition 2.7 (Associativity formula for mixed multiplicities). Let $(R, m)$ be a Noetherian local ring, $q_{1}, \ldots, q_{d}$ m-primary ideals and $M$ a finitely generated $R$-module of dimension $d$. Then

$$
e\left(q_{1}, \ldots, q_{d} ; M\right)=\sum_{p \in \Lambda} \lambda\left(M_{p}\right) e\left(q_{1}, \ldots, q_{d} ; R / p\right)
$$

where $\Lambda=\{p \in \operatorname{Spec}(R): \operatorname{ann}(M) \subseteq p$ and $\operatorname{dim} R / p=\operatorname{dim} M\}$.

Proof. Let $d=\operatorname{dim} M$ and $\Lambda=\left\{p_{1}, \ldots, p_{l}\right\}$. We may choose a sufficiently general element $a_{1} \in q_{i}$ (if $d_{i}>0$ ) as in Theorem 1.7 which will work for the $d$-dimensional modules $R / p_{1}, \ldots, R / p_{l}$ and $M$. We then pass to the $(d-1)$-dimensional modules 
$R /\left(p_{1}+\left(a_{1}\right)\right), \ldots, R /\left(p_{l}+\left(a_{1}\right)\right)$ and $M / a_{1} M$ to choose $a_{2}$, etc. In this way we obtain $a_{1}, \ldots, a_{d}$ for which

$$
\left.e\left(q_{1}, \ldots, q_{d} ; M\right)=e\left(a_{1}, \ldots, a_{d}\right) ; M\right) \text { and } e\left(q_{1}, \ldots, q_{d} ; R / p_{i}\right)=e\left(\left(a_{1}, \ldots, a_{d}\right) ; R / p_{i}\right)
$$

for $i=1, \ldots, l$. Hence

$$
\begin{aligned}
e\left(q_{1}, \ldots, q_{d} ; M\right) & =e\left(\left(a_{1}, \ldots, a_{d}\right) ; M\right)=e\left(\left(a_{1}, \ldots, a_{d}\right)+\operatorname{ann}(M) ; M\right) \\
& =\sum_{i=1}^{l} \lambda\left(M_{p_{\mathfrak{i}}}\right) e\left(\left(a_{1}, \ldots, a_{d}\right)+\operatorname{ann}(M) ; R / p_{\mathfrak{i}}\right),
\end{aligned}
$$

by the usual associativity formula. But also

$$
\begin{aligned}
\sum_{i=1}^{l} \lambda\left(M_{p_{i}}\right) e\left(\left(a_{1}, \ldots, a_{d}\right)+\operatorname{ann}(M) ; R / p_{i}\right) & =\sum_{i=1}^{l} \lambda\left(M_{p_{i}}\right) e\left(\left(a_{1}, \ldots, a_{d}\right) ; R / p_{i}\right) \\
& =\sum_{i=1}^{l} \lambda\left(M_{p_{i}}\right) e\left(q_{1}, \ldots, q_{d} ; R / p_{i}\right) .
\end{aligned}
$$

For a more general version and a very different proof, see [13, Theorem 12.2.7].

LEMMA 2.8. Let $(R, m)$ be a local ring and $M$ a finitely generated $R$-module of dimension $d$. Let $q_{1}, \ldots, q_{d}$ be m-primary and $a_{t} \in q_{i}$ such that $\left(a_{1}, \ldots, a_{d}\right)+\operatorname{ann}(M)$ is m-primary. Then

$$
e\left(\left(a_{1}, \ldots, a_{d}\right) ; M\right) \geqslant e\left(q_{1}, \ldots, q_{d} ; M\right) .
$$

Proof. By Lemma 2.2, there exist $m$-primary ideals $p_{i} \subseteq q_{i}$ such that $a_{i} \in p_{i}$ and $\left(a_{1}, \ldots, a_{d}\right)$ is a joint reduction of $p_{1}, \ldots, p_{d}$ with respect to $R / \operatorname{ann}(M)$, that is with respect to $M$. So $e\left(\left(a_{1}, \ldots, a_{d}\right) ; M\right)=e\left(p_{1}, \ldots, p_{d} ; M\right)$. But by Lemma 2.4 this multiplicity is greater than or equal to $e\left(q_{1}, \ldots, q_{d} ; M\right)$.

The following is a well-known fact about multiplicities.

LEMMA 2.9. The inequality $e_{I}(M) \leqslant e_{I}(M / a M)$ holds for any element $a \in I$ and any finitely generated $R$-module $M$ whenever $\operatorname{dim}(M / a M)<\operatorname{dim}(M)$.

Proof. The exactness of

$$
0 \longrightarrow \frac{I^{n} M:_{M} a}{I^{n-1} M} \longrightarrow \frac{M}{I^{n-1} M} \stackrel{a}{\longrightarrow} \frac{M}{I^{n} M} \longrightarrow \frac{M}{I^{n} M+a M} \longrightarrow 0
$$

gives

$$
\lambda\left(\frac{M}{I^{n} M}\right)-\lambda\left(\frac{M}{I^{n-1} M}\right)=\lambda\left(\frac{M}{I^{n} M+a M}\right)-\lambda\left(\frac{I^{n} M:_{M} a}{I^{n-1} M}\right) .
$$

By the assumption on $a$, each side is a polynomial in $n$ of degree $d-1$ for large $n$. The leading coefficient on the left is $e_{I}(M)$ and the leading coefficient of $\lambda\left(M /\left(I^{n} M+a M\right)\right)$ is $e_{I}(M / a M)$.

\section{Extension of Rees' theorem to mixed multiplicities}

Rees proved in [11] that if $(R, m)$ is a quasi-unmixed local ring, that is its $m$-adic completion is equidimensional, and $I \subseteq J$ are $m$-primary ideals with $e_{I}(R)=e_{J}(R)$, then $I$ is a reduction of $J$. In this section we prove an extension of this to joint reductions and mixed multiplicities. The main theorem is Theorem 3.7, and essential Lemmas are 3.1 and 3.6. 
LEMMA 3.1 (Böger [2]). Let $q_{1}, \ldots, q_{k}$ be ideals in a Noetherian local ring $(R, m)$ with infinite residue field such that $q_{1} \subseteq \sqrt{ }\left(q_{2} \ldots q_{k}\right)$. Let $a_{1} \in q_{1}$. Then there exist integers $c_{0}$ and $l^{0}$ and a sufficiently general element $b \in q_{1}$ such that for all $l \geqslant l^{0}$ and all sufficiently large $n_{1}, \ldots, n_{k}$ (depending on $l$ ),

and

$$
\underline{q}^{\underline{n}} R[Y] \cap\left(a_{1}^{l}+b^{l} Y\right) R[Y]=\left(a_{1}^{l}+b^{l} Y\right) q_{1}^{n_{1}-l} q_{2}^{n_{2}} \ldots q_{k}^{n_{k}} R[Y]
$$

$$
\left(\underline{q}^{\underline{n}} R[Y]:_{R[Y]} a_{1}^{l}+b^{l} Y\right) \cap q_{1}^{c_{0}} q_{2}^{n_{2}} \ldots q_{k}^{n_{k}} R[Y]=q_{1}^{n_{1}-l} q_{2}^{n_{2}} \ldots q_{k}^{n_{k}} R[Y] .
$$

Moreover, if $q_{1}$ does not lie in some given finite set of primes of $R$, then there exists such an element $b$ not contained in any of those primes of $R$.

Proof. Choose an integer $c_{0}$ and a sufficiently general element $b \in q_{1}$ as in Lemma 1.9 with the additional assumption that $b$ does not lie in any associated prime of $(0)$ which does not contain $q_{1}$.

Let $(0)=\bigcap_{i=1}^{s} m_{i}$ be a primary decomposition of (0). Assume that $q_{1} \subseteq \sqrt{ } m_{i}$ for $i=1, \ldots, t$ and that $q_{1} \nsubseteq \sqrt{ } m_{i}$ for $i=t+1, \ldots, s$. Then there exists an integer $l^{0}$ such that for all $l \geqslant l^{0}, q_{1}^{l} \subseteq m_{1} \cap \ldots \cap m_{t}$.

Let $x \in 0: b^{l}$. So $x b^{l}=0 \in m_{t+1} \cap \ldots \cap m_{s}$, so by the choice of $b, x \in m_{t+1} \cap \ldots \cap m_{s}$. Hence $x q_{1}^{l}$ is contained in all ideals $m_{i}$, so $x q_{1}^{l}=0$. Thus $0: b^{l}=0: q_{1}^{l}$ for all $l \geqslant l^{0}$.

ClaIm. For all sufficiently large $n_{1}, \ldots, n_{k}, \underline{q}^{\underline{n}}: b^{l} \subseteq 0: b^{l}+q_{1}^{n_{1}-l} q_{2}^{n_{2}} \ldots q_{k}^{n_{k}}$.

Proof of the claim. By an easy generalization of the Artin-Rees lemma there exists a $k$-tuple $\underline{m} \geqslant \underline{0}$ such that for all $\underline{n} \geqslant \underline{m}$,

$$
b^{l}\left(\underline{q}^{\underline{n}}: b^{l}\right)=\underline{q}^{\underline{n}} \cap\left(b^{l}\right) \subseteq b^{l} \underline{q}^{\underline{n}-\underline{m}} .
$$

Hence $\underline{q}^{\underline{n}}: b^{l} \subseteq 0: b^{l}+\underline{q}^{\underline{n}-\underline{m}}$ and, in fact, $\underline{q}^{\underline{n}}: b^{l} \subseteq 0: b^{l}+\left(\underline{q}^{\underline{n}-\underline{m}} \cap\left(\underline{q}^{\underline{n}}: b^{l}\right)\right)$. But $q_{1} \subseteq \sqrt{ }\left(q_{2} \ldots q_{k}\right)$, so if $n_{1}$ is sufficiently large, $q_{1}^{n_{1}-m_{1}-c_{0}} \subseteq q_{2}^{m_{2}} \ldots \bar{q}_{k}^{m_{k}}$, and so

$$
\begin{aligned}
\underline{q}^{\underline{n}}: b^{l} & \subseteq 0: b^{l}+\left(\underline{q}^{\underline{n}-\underline{m}} \cap\left(q^{\underline{n}}: b^{l}\right)\right) \\
& \subseteq 0: b^{l}+\left(q_{1}^{c_{0}} q_{2}^{n_{2}} \ldots q_{k}^{n_{k}} \cap\left(\underline{q}^{\underline{n}}: b^{l}\right)\right) \\
& =0: q_{1}^{l}+\left(q_{1}^{c_{0}} q_{2}^{n_{2}} \ldots q_{k}^{n_{k}} \cap\left(\underline{q}^{\underline{n}}: b^{l}\right)\right) .
\end{aligned}
$$

Therefore for $\underline{n}$ sufficiently large, by Lemma 1.9, $\underline{q}^{\underline{n}}: b^{l} \subseteq 0: q_{1}^{l}+q_{1}^{n_{1}-l} q_{2}^{n_{2}} \ldots q_{k}^{n_{k}}$.

To show equality in (1), let $F=\left(a_{1}^{l}+b^{l} Y\right)\left(\sum_{j-1}^{s} c_{j} Y^{j}\right)$ be in $\underline{q}^{n} R[Y]$. Then $b^{l} c_{s} \in q^{\underline{n}}$ and $c_{s} \in q^{\underline{n}}: b^{l} \subseteq 0: q_{1}^{l}+q_{1}^{n_{1}-l} q_{2}^{n_{2}} \ldots q_{d}^{n_{d}}$. So $c_{s}=d+e$, where $d \in 0: q_{1}^{l}$ and $e \in q_{1}^{n_{1}-l} q_{2}^{n_{2}} \ldots q_{d}^{n_{d}}$. Hence $F=\left(a_{1}^{l}+b^{l} Y\right)\left(\sum_{j=1}^{s-1} c_{j} Y^{j}+e Y^{s}\right)$. So it suffices to show that

$$
\left(a_{1}^{l}+b^{l} Y\right)\left(\sum_{j=1}^{s-1} c_{j} Y^{j}\right),
$$

an element of $\underline{q}^{n} R[Y]$, belongs to $\left(a_{1}^{l}+b^{l} Y\right) q_{1}^{n_{1}-l} q_{2}^{n_{2}} \ldots q_{d}^{n_{d}} R[Y]$. To show this, just repeat the argument. This proves equality in (1).

If, moreover, each $c_{j}$ lies in $q_{1}^{c_{0}} q_{2}^{n_{2}} \ldots q_{k}^{n_{k}}$, the same argument shows equality in (2).

COROllaRy 3.2. Let $(R, m)$ be a Noetherian local ring with infinite residue field, and let $q_{1}, \ldots, q_{k}$ be ideals. Let $a_{i} \in q_{i}$ for $i=1, \ldots, k$. Assume that $\left(a_{1}, \ldots, a_{k}\right)$ has the same radical as all the $q_{i}$ and that the common height of these ideals is $k$. If $p$ is a prime, minimal over $\left(a_{1}, \ldots, a_{k}\right)$, such that $e\left(\left(a_{1}, \ldots, a_{k}\right) R_{p} ; R_{p}\right)=e\left(q_{1} R_{p}, \ldots, q_{k} R_{p} ; R_{p}\right)$, then there exists a sufficiently general element $b \in q_{1}$ such that for all sufficiently large integers $l$,

$$
e\left(\left(a_{1}, \ldots, a_{k}\right) R_{p} ; R_{p}\right)=(1 / l) e\left(\left(a_{1}^{l}+b^{l} Y, a_{2}, \ldots, a_{k}\right) R[Y]_{p R[Y]} ; R[Y]_{p R[Y]}\right) .
$$


Proof by induction on $k$. If $k=1$, we may choose $b$ sufficiently general in $q_{1}$ as in Lemma 3.1 which is not contained in any minimal prime of $R$. Then for all $l \geqslant l^{0}$, $a_{1}^{l}+b^{l} Y$ is a superficial element for $q_{1}^{l} R[Y]_{p R[Y]}$ and the module $R[Y]_{p R[Y]}$. Hence

$$
\begin{aligned}
e\left(\left(a_{1}^{l}+b^{l} Y\right) ; R[Y]_{p R[Y]}\right) & =e\left(q_{1}^{l} R[Y]_{p R[Y]} ; R[Y]_{p R[Y]}\right) \quad \text { (by Theorem 1.11) } \\
& =e\left(q_{1}^{l} R_{p} ; R_{p}\right) \quad\left(\text { since } \lambda\left(R / q_{1}^{n}\right)_{p}=\lambda\left(\left(R / q_{1}^{n}[Y]\right)_{p R[Y]}\right)\right) \\
& =l e\left(q_{1} R_{p} ; R_{p}\right) \quad(\text { by Lemma 2.6), }
\end{aligned}
$$

which finishes the proof for the case $k=1$.

Now suppose that $k \geqslant 2$ and that the corollary is true for $k-1$. First suppose that the corollary is known for domains. Let $\Lambda$ be the set of all minimal primes $Q$ of $R$ lying inside $p$ such that $\operatorname{dim}\left(R_{p}\right)=\operatorname{dim}\left((R / Q)_{p}\right)$. By the associativity formulas for usual and mixed multiplicities and by Lemma 2.8 , the assumptions are still true in $R / Q$ for every $Q \in \Lambda$. Fix $Q \in \Lambda$ and let $S_{Q}=(R[Y] / Q R[Y])_{p R[Y]}$. Since the result is true for domains, there exists a sufficiently general element $b$ in $q_{1}+Q / Q$ such that

$$
e\left(\left(a_{1}, \ldots, a_{k}\right) S_{Q} ; S_{Q}\right)=(1 / l) e\left(\left(a_{1}^{l}+b^{l} Y, a_{2}, \ldots, a_{k}\right) S_{Q} ; S_{Q}\right)
$$

for all sufficiently large integers $l$. By lifting $b$ to $R$ we get a sufficiently general element of $q_{1}$ for which the above equation is true. Then since $\Lambda$ is finite, we get a sufficiently general element $b \in q_{1}$ such that the above equation holds for all sufficiently large integers $l$ and all $Q \in \Lambda$. Then by using associativity formulas again we get

$$
\begin{aligned}
e\left(\left(a_{1}, \ldots, a_{k}\right) R_{p} ; R_{p}\right) & =\sum_{Q \in \Lambda} \lambda\left(R_{Q}\right) e\left(\left(a_{1}, \ldots, a_{k}\right) R_{p} ;(R / Q)_{p}\right) \\
& =\sum_{Q \in \Lambda} \lambda\left(R_{Q}\right) e\left(\left(a_{1}, \ldots, a_{k}\right) S_{Q} ; S_{Q}\right) \\
& =\sum_{Q \in \Lambda} \lambda\left(R_{Q}\right) \frac{1}{l} e\left(\left(a_{1}^{l}+b^{l} Y, a_{2}, \ldots, a_{k}\right) S_{Q} ; S_{Q}\right) \\
& =(1 / l) e\left(\left(a_{1}^{l}+b^{l} Y, a_{2}, \ldots, a_{k}\right) R[Y]_{p R[Y]} ; R[Y]_{p R[Y]}\right),
\end{aligned}
$$

as desired. So we have only to show the existence of such sufficiently general elements for domains. In this case $a_{k}$ is not a zero divisor on $R$.

ClAIM. $e\left(\left(a_{1}, \ldots, a_{k-1}\right) R_{p} ;\left(R /\left(a_{k}\right)\right)_{p}\right)=e\left(q_{1} R_{p}, \ldots, q_{k-1} R_{p} ;\left(R /\left(a_{k}\right)\right)_{p}\right)$.

Proof. Let $S=R[Y]_{p R[Y]}$ which has an infinite residue field. Then

$$
\begin{aligned}
e\left(q_{1} R_{p}, \ldots, q_{k} R_{p} ; R_{p}\right) & =e\left(\left(a_{1}, \ldots, a_{k}\right) R_{p} ; R_{p}\right) \\
& =e\left(\left(a_{1}, \ldots, a_{k-1}\right) R_{p} ;\left(R /\left(a_{k}\right)\right)_{p}\right) \quad \text { (by [5, Theorem 14.11]) } \\
& \geqslant e\left(q_{1} R_{p}, \ldots, q_{k-1} R_{p} ;\left(R /\left(a_{k}\right)\right)_{p}\right)
\end{aligned}
$$

(by Lemma 2.8 since $a_{i} \in q_{i}$ )

$$
\begin{aligned}
& =e\left(q_{1} S, \ldots, q_{k-1} S ; S /\left(a_{k}\right)\right) \\
& =e\left(\left(b_{1}, \ldots, b_{k-1}\right) S ; S /\left(a_{k}\right)\right)
\end{aligned}
$$

(by Theorems 1.9 and 1.11 for some $b_{i} \in q_{i} S$ )

$$
\begin{aligned}
& =e\left(\left(b_{1}, \ldots, b_{k-1}, a_{k}\right) S ; S\right) \quad(\text { by }[5, \text { Theorem } 14.11]) \\
& \geqslant e\left(q_{1} S, \ldots, q_{k} S ; S\right) \quad\left(\text { by Lemma } 2.8 \text { since } b_{i} \in q_{\imath} S, a_{k} \in q_{k}\right) \\
& =e\left(q_{1} R_{p}, \ldots, q_{k} R_{p} ; R_{p}\right),
\end{aligned}
$$

so we have equality throughout, which proves the claim. 
Then by induction there exists a sufficiently general element $b \in q_{1} R /\left(a_{k}\right)$ such that

$$
e\left(\left(a_{1}, \ldots, a_{k-1}\right) R_{p} ;\left(R /\left(a_{k}\right)\right)_{p}\right)=\frac{1}{l} e\left(\left(a_{1}^{l}+b^{l} Y, a_{2}, \ldots, a_{k-1}\right) ;\left(R[Y] /\left(a_{k}\right)\right)_{p R[Y]}\right) .
$$

Since $q_{1} / m q_{1} \rightarrow\left(q_{1}+\left(a_{k}\right)\right) /\left(m q_{1}+\left(a_{k}\right)\right)$ is surjective, $b$ is also a sufficiently general element of $q_{1} R$. Moreover,

$$
\begin{aligned}
e\left(\left(a_{1}, \ldots, a_{k}\right) R_{p} ; R_{p}\right) & =e\left(\left(a_{1}, \ldots, a_{k-1}\right) R_{p} ;\left(R /\left(a_{k}\right)\right)_{p}\right) \\
& =(1 / l) e\left(\left(a_{1}^{l}+b^{l} Y, a_{2}, \ldots, a_{k-1}\right) ;\left(R[Y] /\left(a_{k}\right)\right)_{p R[Y]}\right) \\
& =(1 / l) e\left(\left(a_{1}^{l}+b^{l} Y, a_{2}, \ldots, a_{k}\right) ; R[Y]_{p R[Y]}\right)
\end{aligned}
$$

by [ 5 , Theorem 14.11], which finishes the corollary.

ReMARK 3.3. If the hypotheses of Corollary 3.2 are satisfied for every prime ideal $p$ minimal over $\left(a_{1}, \ldots, a_{k}\right)$, then since there are only finitely many such ideals we get a sufficiently general element $b \in q_{1}$ such that the conclusion of the corollary is true for all such primes $p$ and all sufficiently large integers $l$.

Now we need a definition and a few remarks.

Definition 3.4. A ring $R$ satisfies the first chain condition if $\operatorname{dim}(R)=$ $\operatorname{dim}(R / I)+$ ht $(I)$ for each ideal $I$ in $R$.

REMARK 3.5. If $R$ is quasi-unmixed, then $R$ satisfies the first chain condition and is equidimensional [6,34.5]. Every localization of a polynomial ring over $R$ is still quasi-unmixed [9, Theorem 3.6]. Also, if $a$ is part of a system of parameters of $R$, then $R /(a)$ is quasi-unmixed and the integral closure of $(a)$ has no embedded primes [10, Theorem 2.12].

One can prove Theorem 3.7 by using an argument similar to Böger's, namely by reducing the problem to a zero-dimensional ring. It is not necessary to make such a ( $d$-dimensional) leap, though. It suffices to just drop the dimension by one, which seems more instructive in that it is not as far removed from the original problem. We shall use induction on the number of ideals. For this we need a way to reduce dimension, which is provided by the following lemma.

Lemma 3.6. Let $(R, m)$ be a quasi-unmixed Noetherian local ring with infinite residue field, and let $q_{1}, \ldots, q_{k}$ be ideals. Let $a_{i} \in q_{i}$ for $i=1, \ldots, k$. Assume that $\left(a_{1}, \ldots, a_{k}\right)$ and the $q_{i}$ have the same radical and that their common height is $k$. Let $\Lambda$ be the set of all minimal primes over $\left(a_{1}, \ldots, a_{k}\right)$. Assume that for each $p \in \Lambda$,

$$
e\left(\left(a_{1}, \ldots, a_{k}\right) R_{p} ; R_{p}\right)=e\left(q_{1} R_{p}, \ldots, q_{k} R_{p} ; R_{p}\right) .
$$

Let $b$ be a sufficiently general element of $q_{1}$ as in Remark 3.3 with the additional restriction that $b$ does not lie in any prime of $R$ minimal over $\left(a_{2}, \ldots, a_{k}\right)$. Then for all sufficiently large integers $l$, the set primes of $R[Y]_{(m+(Y)) R[Y]}$ minimal over the ideal $\left(a_{1}^{l}+b^{l} Y, a_{2}, \ldots, a_{k}\right) R[Y]_{(m+(Y)) R[Y]}$ is $\left\{p R[Y]_{(m+(Y)) R[Y]}: p \in \Lambda\right\}$. 
Proof. Let $S=R[Y]_{(m+(Y)) R[Y]}$. By the choice of $\mathrm{b}$, the height of

$$
\left(a_{1}^{l}+b^{l} Y, a_{2}, \ldots, a_{k}\right) S
$$

equals $k$. Clearly elements of $\Lambda$ extend to primes of $S$ minimal over

$$
\left(a_{1}^{l}+b^{l} Y, a_{2}, \ldots, a_{k}\right) S .
$$

Suppose that there exists a prime $Q$, minimal over $\left(a_{1}^{l}+b^{l} Y, a_{2}, \ldots, a_{k}\right) S$, which is not an extension of any $p$ in $\Lambda$. We have ht $(Q) \leqslant k$ and

$$
\operatorname{dim}(S / Q) \leqslant \operatorname{dim}\left(S /\left(a_{1}^{l}+b^{l} Y, a_{2}, \ldots, a_{k}\right) S\right)=\operatorname{dim}(R)+1-k .
$$

Since $S$ satisfies the first chain condition then

$$
\operatorname{dim}(S)=\operatorname{dim}(S / Q)+\mathrm{ht}(Q) \leqslant \operatorname{dim}(R)+1-k+k=\operatorname{dim}(R)+1=\operatorname{dim}(S),
$$

so necessarily $\operatorname{dim}(S / Q)=\operatorname{dim}(R)-k+1$. Similarly, $\operatorname{dim}(S / p S)=\operatorname{dim}(R)-k+1$ for all $p \in \Lambda$. By the associativity formula then

$$
\begin{aligned}
e\left(\frac{S}{\left(\left(a_{1}^{l}+b^{l} Y\right)^{n}, a_{2}^{n}, \ldots, a_{k}^{n}\right) S}\right) \geqslant & e\left(\frac{S}{Q}\right) \lambda\left(\frac{S_{Q}}{\left(\left(a_{1}^{l}+b^{l} Y\right)^{n}, a_{2}^{n}, \ldots, a_{k}^{n}\right) S_{Q}}\right) \\
& +\sum_{p \in \Lambda} e\left(\frac{S}{p S}\right) \lambda\left(\frac{S_{p S}}{\left(\left(a_{1}^{l}+b^{l} Y\right)^{n}, a_{2}^{n}, \ldots, a_{k}^{n}\right) S_{p S}}\right)
\end{aligned}
$$

for all $n \geqslant 1$. So

$$
\begin{aligned}
\lim _{n \rightarrow \infty} & \frac{1}{n^{k}} e\left(\frac{S}{\left(\left(a_{1}^{l}+b^{l} Y\right)^{n}, a_{2}^{n}, \ldots, a_{k}^{n}\right) S}\right) \\
\geqslant & \lim _{n \rightarrow \infty} \frac{1}{n^{k}} e\left(\frac{S}{Q}\right) \lambda\left(\frac{S_{Q}}{\left(\left(a_{1}^{l}+b^{l} Y\right)^{n}, a_{2}^{n}, \ldots, a_{k}^{n}\right) S_{Q}}\right) \\
& \quad+\lim _{n \rightarrow \infty} \frac{1}{n^{k}} \sum_{p \in \Lambda} e\left(\frac{S}{p S}\right) \lambda\left(\frac{S_{p S}}{\left(\left(a_{1}^{l}+b^{l} Y\right)^{n}, a_{2}^{n}, \ldots, a_{k}^{n}\right) S_{p S}}\right) \\
= & e(S / Q) e\left(\left(a_{1}^{l}+b^{l} Y, a_{2}, \ldots, a_{k}\right) S_{Q} ; S_{Q}\right) \\
& +\sum_{p \in \Lambda} e(S / p S) e\left(\left(a_{1}^{l}+b^{l} Y, a_{2}, \ldots, a_{k}\right) S_{p S} ; S_{p S}\right) \quad \text { (by Lech's formula) } \\
> & \sum_{p \in \Lambda} e(S / p S) e\left(\left(a_{1}^{l}+b^{l} Y, a_{2}, \ldots, a_{k}\right) S_{p S} ; S_{p S}\right) .
\end{aligned}
$$

By Lemma 2.9 and the fact that $\left((m+(Y))^{n} S: Y\right)=(m+(Y))^{n-1} S$ for each $n$, $e(S / p S)=e(R / p)$. So by the choice of $b$ (see Corollary 3.2),

$$
\begin{aligned}
\lim _{n \rightarrow \infty} \frac{1}{n^{k}} e\left(\frac{S}{\left(\left(a_{1}^{l}+b^{l} Y\right)^{n}, a_{2}^{n}, \ldots, a_{k}^{n}\right) S}\right) & >\sum_{p \in \Lambda} e(S / p S) e\left(\left(a_{1}^{l}+b^{l} Y, a_{2}, \ldots, a_{k}\right) S_{p S} ; S_{p S}\right) \\
& =\sum_{p \in \Lambda} e(R / p) l e\left(\left(a_{1}, \ldots, a_{k}\right) S_{p S} ; S_{p S}\right) \\
& =\sum_{p \in \Lambda} e(R / p) l e\left(\left(a_{1}, \ldots, a_{k}\right) R_{p} ; R_{p}\right)
\end{aligned}
$$


However, by Lemma 2.9,

$$
\begin{aligned}
e\left(\frac{S}{\left(\left(a_{1}^{l}+b^{l} Y\right)^{n}, a_{2}^{n}, \ldots, a_{k}^{n}\right) S}\right) & \leqslant e\left(\frac{S}{\left(\left(a_{1}^{l}+b^{l} Y\right)^{n}, a_{2}^{n}, \ldots, a_{k}^{n}, Y\right) S}\right) \\
& =e\left(\frac{R}{\left(a_{1}^{l n}, a_{2}^{n}, \ldots, a_{k}^{n}\right)}\right)
\end{aligned}
$$

for all $n$. Since $R$ satisfies the first chain condition,

$$
\operatorname{dim}(R / p)=\operatorname{dim}(R)-\mathrm{ht}(p)=\operatorname{dim}(R)-k=\operatorname{dim}\left(R /\left(a_{1}, \ldots, a_{k}\right)\right)
$$

for every $p \in \Lambda$. By the associativity formula then

$$
e\left(\frac{R}{\left(a_{1}^{l n}, a_{2}^{n}, \ldots, a_{k}^{n}\right)}\right) \leqslant \sum_{p \in \Lambda} e(R / p) \lambda\left(\frac{R}{\left(a_{1}^{l n}, a_{2}^{n}, \ldots, a_{k}^{n}\right) R_{p}}\right),
$$

so by Lech's formula,

$$
\lim _{n \rightarrow \infty} \frac{1}{n^{k}} e\left(\frac{S}{\left(\left(a_{1}^{l}+b^{l} Y\right)^{n}, a_{2}^{n}, \ldots, a_{k}^{n}\right) S}\right) \leqslant \sum_{p \in \Lambda} e(R / p) l e\left(\left(a_{1}, \ldots, a_{k}\right) R_{p} ; R_{p}\right),
$$

contradicting equation (3).

Corollary 3.2 and Lemma 3.6 provide a method of going modulo superficial elements and lifting the result back to the original ring. This is used in the following main theorem of this paper.

THEOREM 3.7. Let $(R, m)$ be a quasi-unmixed Noetherian local ring, let $q_{1}, \ldots, q_{k}$ be ideals and $a_{i}$ an element of $q_{i}$, for $i=1, \ldots, k$. Suppose that the radical of $\left(a_{1}, \ldots, a_{k}\right)$ is the same as the radical of all the $q_{i}$, and that their common height is $k$. If

$$
e\left(\left(a_{1}, \ldots, a_{k}\right) R_{p} ; R_{p}\right)=e\left(q_{1} R_{p}, \ldots, q_{k} R_{p} ; R_{p}\right)
$$

for each prime ideal $p$ minimal over $\left(a_{1}, \ldots, a_{k}\right)$, then $\left(a_{1}, \ldots, a_{k}\right)$ is a joint reduction of $\left(q_{1}, \ldots, q_{k}\right)$.

Proof by induction on $k$. If $k=0$, there is nothing to prove.

Let $\Lambda$ be the set of all primes minimal over $\left(a_{1}, \ldots, a_{k}\right)$. Let $S=R[X]_{m R[X]}$ which has an infinite residue field; $S$ is still a quasi-unmixed local ring. Since

$$
\lambda\left(R_{p} / I R_{p}\right)=\lambda\left(S_{p s} / I S_{p S}\right)
$$

for any $p \in \Lambda$ and any ideal $I$ such that $p$ is minimal over $I$, it is easy to see that all the hypotheses are still satisfied on $S$. Also, by faithful flatness of $S$ over $R$ the conclusion is true in $R$ if and only if it is true in $S$, so without loss of generality assume that the residue field of $R$ is infinite.

By the associativity formulas and by Lemma 2.8 we get that

$$
e\left(\left(a_{1}, \ldots, a_{k}\right) R_{p} ; R_{p} / Q R_{p}\right)=e\left(q_{1} R_{p}, \ldots, q_{k} R_{p} ; R_{p} / Q R_{p}\right)
$$

for every minimal prime $Q$ of $R$ and for every $p \in \Lambda$. Notice that each $R / Q$ is a quasiunmixed domain. Set $I=a_{1} q_{2} \ldots q_{k}+\ldots+a_{k} q_{1} \ldots q_{k-1}$. If the theorem is true for domains, then we get that $I$ is a reduction of $q_{1} \ldots q_{k}$ modulo each minimal prime of $R$. Hence by [11, Lemma 1.2], $I$ is a reduction of $q_{1} \ldots q_{k} R$, which finishes the theorem. So it is enough to prove the theorem for domains. 
If $k=1$, by assumption $e\left(\left(a_{1}\right) R_{p} ; R_{p}\right)=e\left(q_{1} R_{p} ; R_{p}\right)$ for every $p \in \Lambda$, so

$$
\begin{aligned}
\lambda\left(R_{p} / q_{1}^{n} R_{p}\right)-\lambda\left(R_{p} / q_{1}^{n-1} R_{p}\right) & =e\left(q_{1} R_{p} ; R_{p}\right) \quad \text { (for } n \text { sufficiently large) } \\
& =e\left(\left(a_{1}\right) R_{p} ; R_{p}\right) \\
& =\lambda\left(R_{p} /\left(a_{1}\right) R_{p}\right) \quad \text { (since } a_{1} \text { is not a zero divisor) } \\
& =\lambda\left(R_{p} / a_{1} q_{1}^{n-1} R_{p}\right)-\lambda\left(\left(a_{1}\right) R_{p} / a_{1} q_{1}^{n-1} R_{p}\right) \\
& =\lambda\left(R_{p} / a_{1} q_{1}^{n-1} R_{p}\right)-\lambda\left(R_{p} / q_{1}^{n-1} R_{p}\right) .
\end{aligned}
$$

So $\lambda\left(R_{p} / q_{1}^{n} R_{p}\right)=\lambda\left(R_{p} / a_{1} q_{1}^{n-1} R_{p}\right)$, and since $a_{1} q_{1}^{n-1} \subseteq q_{1}^{n}$, necessarily $a_{1} q_{1}^{n-1} R_{p}=q_{1}^{n} R_{p}$. This means that the integral closure $\overline{\left(a_{1}\right)}$ of $\left(a_{1}\right)$ is the same as $\bar{q}_{1}$ after localization at every $p \in \Lambda$. Then by Remark 3.5, since $\overline{\left(a_{1}\right)}$ has no embedded primes, $\overline{\left(a_{1}\right)}=\overline{q_{1}}$. Hence $\left(a_{1}\right)$ is a (joint) reduction of $q_{1}$.

Now assume that $k \geqslant 2$. By Corollary 3.2 there exists a sufficiently general element $b \in q_{1}$ such that for all sufficiently large integers $l$,

$$
e\left(\left(a_{1}, \ldots, a_{k}\right) R_{p} ; R_{p}\right)=(1 / l) e\left(\left(a_{1}^{l}+b^{l} Y, a_{2}, \ldots, a_{k}\right) R[Y]_{p R[Y]} ; R[Y]_{p R[Y]}\right)
$$

and

$$
\left.e\left(q_{1}^{l} R_{p}, q_{2} R_{p}, \ldots, q_{k} R_{p} ; R_{p}\right)=e\left(q_{2} R_{p}, \ldots, q_{k} R_{p} ;\left(R[Y] / a_{1}^{l}+b^{l} Y\right)\right)_{p R[Y]}\right)
$$

for every $p$ in $\Lambda$. (For the last equality, Theorem 1.11 is also needed.)

Set $S=\left(R[Y] /\left(a_{1}^{l}+b^{l} Y\right)\right)_{(m+(Y)) R[Y]}$. Then $S$ is quasi-unmixed by Remark 3.5 and

$$
\begin{aligned}
e\left(\left(a_{2}, \ldots, a_{k}\right) S_{p S} ; S_{p S}\right) & =e\left(\left(a_{1}^{l}+b^{l} Y, a_{2}, \ldots, a_{k}\right) R[Y]_{p R[Y]} ; R[Y]_{p R[Y]}\right) \\
& =l e\left(\left(a_{1}, \ldots, a_{k}\right) R_{p} ; R_{p}\right)=l e\left(q_{1} R_{p}, \ldots, q_{k} R_{p} ; R_{p}\right) \\
& =e\left(q_{1}^{l} R_{p}, q_{2} R_{p}, \ldots, q_{k} R_{p} ; R_{p}\right)=e\left(q_{2} S_{p S}, \ldots, q_{k} S_{p S} ; S_{p S}\right)
\end{aligned}
$$

for every $p \in \Lambda$. By Lemma 3.6 and by induction then $\left(a_{2}, \ldots, a_{k}\right)$ is a joint reduction of $\left(q_{2} S, \ldots, q_{k} S\right)$. Set $J=a_{2} q_{3} \ldots q_{k}+\ldots+a_{k} q_{2} \ldots q_{k-1}$. Then the above means that $J S$ is a reduction of $q_{2} \ldots q_{k} S$, that is $\left(q_{2} \ldots q_{k}\right)^{n} S \subseteq J\left(q_{2} \ldots q_{k}\right)^{n-1} S$ for all sufficiently large integers $n$. This means that

$$
\left(q_{2} \ldots q_{k}\right)^{n} T \subseteq J\left(q_{2} \ldots q_{k}\right)^{n-1} T+\left(a_{1}^{l}+b^{l} Y\right) \cap\left(q_{2} \ldots q_{k}\right)^{n} T,
$$

where $T=R[Y]_{(+(Y)) R[Y]}$. By the choice of $b$ as in Lemma 3.1 then, for possibly larger $n$,

$$
\left(q_{1} \ldots q_{k}\right)^{n} T \subseteq J q_{1}\left(q_{1} \ldots q_{k}\right)^{n-1} T+\left(a_{1}^{l}+b^{l} Y\right) q_{1}^{n-l}\left(q_{2} \ldots q_{k}\right)^{n} T .
$$

So there exists $s$ in $R[Y]$, not contained in $(m+(Y)) R[Y]$, such that

$$
s\left(q_{1} \ldots q_{k}\right)^{n} R[Y] \subseteq J q_{1}\left(q_{1} \ldots q_{k}\right)^{n-1} R[Y]+\left(a_{1}^{l}+b^{l} Y\right) q_{1}^{n-l}\left(q_{2} \ldots q_{k}\right)^{n} R[Y] .
$$

Since $s$ contains a term $u$ which is a unit in $R$, by reading off the degree zero monomials we have that

$$
\begin{aligned}
\left(q_{1} \ldots q_{k}\right)^{n} & =u\left(q_{1} \ldots q_{k}\right)^{n} \\
& \subseteq J q_{1}\left(q_{1} \ldots q_{k}\right)^{n-1}+a_{1}^{l} q_{1}^{n-l}\left(q_{2} \ldots q_{k}\right)^{n} \\
& \subseteq J q_{1}\left(q_{1} \ldots q_{k}\right)^{n-1}+a_{1} q_{1}^{n-1}\left(q_{2} \ldots q_{k}\right)^{n},
\end{aligned}
$$

so that $\left(a_{1}, \ldots, a_{k}\right)$ is a joint reduction of $\left(q_{1}, \ldots, q_{k}\right)$.

COROLlary 3.8 (Rees [11]). Let $(R, m)$ be a quasi-unmixed local ring and $I \subseteq J$ m-primary ideals. If $e_{I}(R)=e_{J}(R)$, then $I$ is a reduction of $J$. 
Proof. As in the proof of Theorem 3.7 assume that $|R / m|=\infty$. Let $d=\operatorname{dim}(R)$. Then by a result of Northcott and Rees [7] there exist elements $a_{1}, \ldots, a_{d} \in I$ such that $\left(a_{1}, \ldots, a_{d}\right)$ is a reduction of $I$. So $e\left(\left(a_{1}, \ldots, a_{d}\right), R\right)=e_{I}(R)=e_{J}(R)$. By Lemma 2.5, $e_{J}(R)$ equals $e(J, \ldots, J ; R)$, so by the previous theorem $\left(a_{1}, \ldots, a_{d}\right)$ is a joint reduction of $(J, \ldots, J)$. This means that the ideal $\left(a_{1}, \ldots, a_{d}\right)$ is a reduction of $J$, so a fortiori $I$ is a reduction of $J$.

COROLLARY 3.9 (Böger [2]). Let $I \subseteq J \subseteq \sqrt{ } I$ be ideals in a quasi-unmixed local ring. Assume that the analytic spread of I equals the height of $I$. Then $I$ is a reduction of $J$ if $e\left(I R_{p} ; R_{p}\right)=e\left(J R_{p} ; R_{p}\right)$ for all primes $p$ minimal over $I$.

Proof. Again we may assume that the residue field is infinite, so that there exists a reduction $\left(a_{1}, \ldots, a_{k}\right)$ of $I$, where $k$ is the analytic spread of $I$. For each prime $p$ minimal over $I, e\left(\left(a_{1}, \ldots, a_{k}\right) R_{p} ; R_{p}\right)=e\left(I R_{p} ; R_{p}\right)=e\left(J R_{p} ; R_{p}\right)$, so by Theorem 3.7 , $\left(a_{1}, \ldots, a_{k}\right)$ is a joint reduction of $(J, \ldots, J)$. This means that $\left(a_{1}, \ldots, a_{k}\right)$ is a reduction of $J$, and a fortiori $I$ is a reduction of $J$.

Acknowledgement. I thank Craig Huneke for many conversations regarding this paper.

\section{References}

1. P. B. Bhattacharya, 'The Hilbert function of two ideals', Proc. Cambridge Philos. Soc. 53 (1957) 568-575.

2. E. BöGER, 'Eine Verallgemeinerung eines Multiplizitätensatzes von D. Rees', J. Algebra 12 (1969) 207-215.

3. D. Katz, 'Note on multiplicity', Proc. Amer. Math. Soc. 104 (1988) 1021-1026.

4. D. KIRBY and D. REES, 'Hilbert functions of multigraded modules and the Buchsbaum-Rim multiplicity’, preprint, 1992.

5. H. Matsumura. Commutative ring theory (University Press, Cambridge, 1986).

6. M. Nagata. Local rings (John Wiley, New York, 1962).

7. D. G. NorthCotT and D. ReEs, 'Reductions of ideals in local rings', Proc. Cambridge Philos. Soc. 50 (1954) 145-158.

8. L. O'Carroll, 'On two theorems concerning reductions in local rings', J. Math. Kyoto Univ. 27 (1987) 61-67.

9. L. J. RAtLIFF, JR, 'On quasi-unmixed local domains, the altitude formula, and the chain condition for prime ideals, (i)', Amer. J. Math. 91 (1969) 508-528.

10. L.J. RatlifF, $\mathbf{J}_{\mathrm{R}}$, 'Locally quasi-unmixed noetherian rings and ideals of the principal class', Pacific J. Math. 52 (1974) 185-205.

11. D. ReEs, ' $A$-transforms of local rings and a theorem on multiplicities of ideals', Proc. Cambridge Philos. Soc. 57 (1961) 8-17.

12. D. REES, 'Generalizations of reductions and mixed multiplicities', J. London Math. Soc. 29 (1984) 397-414.

13. D. REES, Lectures on the asymptotic theory of ideals (University Press, Cambridge, 1988).

14. D. ReEs and R. Y. SHARP, 'On a theorem of B. Teissier on multiplicities of ideals in local rings', J. London Math. Soc. (2) 18 (1978) 449-463.

15. B. Teissier, 'Cycles évanescents, sections planes, et conditions de Whitney', Singularités à Cargèse (1972) 285-362, Astérisque 7-8 (1973).

16. J. K. Verma, 'A criterion for joint reductions', note, 1987.

17. J. K. Verma, 'Rees algebras and mixed multiplicities', Proc. Amer. Math. Soc. 104 (1988) 1036-1044.

18. J. K. Verma, 'Joint reductions of complete ideals', Nagoya Math. J. 118 (1990) 155-163.

Department of Mathematics

University of Michigan

Ann Arbor

Michigan 48109-1003

USA 